\title{
How do People Make Decisions with/without Smartphones While Walking in the City?
}

\author{
Kaori Ito ${ }^{\text {a, }}$, Asahi Maeda ${ }^{\text {a }}$, Kota Katsumata ${ }^{\text {, }}$, Seiya Takayanagi ${ }^{\text {a }}$ \\ ${ }^{a}$ Department of Architecture, Faculty of Science and Technology, Tokyo University of Science, kaori@rs.tus.ac.jp \\ ${ }^{b}$ Fujitsu Limited \\ * Corresponding author
}

Keywords: Decision-making, Map application, Smartphone, Visited places, Urban environment

\begin{abstract}
:
The experience of walking in the city has changed since the diffusion of smartphones with information communication and GNSS-based navigation technologies. In our previous work (Ito et al., 2019), we conducted an experiment in which each participating student walked freely by him-/herself with his/her smartphone, classified them by sketch map types, and showed their senses of direction and use of map application varied by sketch map type.

In this manuscript, we focus on participants' decision-making while walking in the city. Our research objective is to investigate the difference among decision-making with smartphones and those with other sources such as accidental passer-by and his/her memory of the past experiences. The significance of the study is that the impact of smartphone use on how people contact a real urban environment is examined through experiments of walking freely.

We organized experiments in which students were asked to walk by him-/herself from a given starting point to a final destination. The participants were told that they did not need to follow the direct route to the final destination and could visit any places in the neighborhood as long as they travelled on foot and arrived at the final destination before the sunset. Each participant walked with his/her own smartphone and used it as usual. Their use of smartphones was recorded within their permissions in order to record what application they used and how they used it. Additionally, participants were interviewed about their decision-making following the experiment.
\end{abstract}

We will show the results of multiple comparison tests to determine the relationships among sources of decision-making, location and type of visiting places, and map app uses.

\section{Acknowledgements:}

The study was supported by JSPS KAKENHI Grant Number JP17H00839.

\section{References:}

Kaori Ito, Yusuke Sakurai, Yuri Fujita, Andrew Burgess (2019), A note on the Relationship between Spatial Recognition and Behavior while Walking in the City, The 29th International Cartographic Conference Abstracts of the ICA. 